\title{
ANGUS: A New Approach to Three-Dimensional Reconstruction of Coronary Vessels by Combined Use of Angiography and Intravascular Ultrasound
}

\author{
M Laban, JA Oomen, CJ Slager, JJ Wentzel, R Krams, JCH Schuurbiers, A den Boer, \\ $\mathrm{C}$ von Birgelen, PW Serruys, PJ de Feijter
}

Thorax Center, University Hospital Rotterdam, Netherlands

\begin{abstract}
Current three dimensional (3D) vessel reconstruction using intravascular ultrasound (IVUS) pull back is limited by the lack of information on the real vessel curvatures, because the movement of the catheter is assumed to proceed along a straight path. To overcome this limitation a method (ANGUS) has been developed to combine coronary angiography with data obtained by IVUS [1].

The IVUS data represent a cylindrical stack of crosssections. A least-square-fit approximation is used to reconstruct the $3 D$ path of the catheter axis from two biplane X-ray images, after which the stack of IVUS contours is wrapped around this $3 D$ catheter centerline. In order to establish the correct rotational position of the stack around the centerline, use is made of 'landmarks' which are visible in angiograms, as well as in a simulation of these angiograms derived from the reconstructed $3 D$ contour.

The combination of ANGiography and intravascular UltraSound (ANGUS) is promising and provides a unique method of three-dimensional reconstruction of coronary geometry.
\end{abstract}

\section{Introduction}

From a sequence of IVUS images, acquired during automated, constant-speed pull back, a 3D image of a coronary artery can be reconstructed, by stacking the acquired frames along a straight axis. This kind of reconstruction is extensively described in the literature [2] and some commercial available IVUS systems have such a possibility build in. However, in these images the straight orientation of the vessel segment is a simplification, because the catheter tip will in reality traverse a spatial curve, the effect of which is hidden from the observer. To improve on this subject we added a method to reconstruct the spatial curve traversed by the catheter tip using a biplane X-ray image of the catheter at the start of the pull back procedure.

We apply, as a first step, the same IVUS technology during an automated pull back of a sheathed IVUS catheter at a speed of $1 \mathrm{~mm} / \mathrm{sec}$. Start of the pull-back procedure is recorded by biplane $\mathrm{X}$-ray. The IVUS catheter tip will follow the sheath closely during pull back and the sheath itself will keep its relative position within the artery during this time. At the completion of the procedure biplane coronary angiography is performed without any change in geometrical $\mathrm{X}$-ray settings. The biplane X-ray images, showing the catheter position shortly after start of pull back, allow geometrical reconstruction of the position of the catheter's longitudinal centerline, which is assumed to represent the path of the catheter tip during pull-back.

From the IVUS images end diastolic samples are selected and from these the contours of the lumen wall and external elastic membrane are semi-automatically detected [3]. These contours are stacked and smoothed by removal of the higher frequency components using a 2 dimensional Fourier transform method.

Finally, these IVUS contours are combined with the reconstructed path of the catheter tip using the features of the angiograms to establish the rotational position of the IVUS contours around the spatial curve.

\section{Methods}

\subsection{Processing the IVUS images}

The IVUS images are recorded during automated pull back on a PAL standard S-VHS video tape, resulting in 25 images/second. A few seconds after pull back has started a short biplane X-ray film is made and the start of this film is marked on the audio track of the S-VHS tape to enable synchronization with the IVUS contours sequence. The IVUS picture to be selected is augmented with a marker which indicates the occurrence of the R-peak in the patient's ECG.

By filming the position of the catheter shortly after start of pull back, any backlash that the automatic pull back system might experience has disappeared and a precise relation between the echo image location in the filmed biplane images and the recorded IVUS pictures can be established. This is done by locating the ultrasound crystal as seen in both of the biplane film images.

Following the acquisition, the S-VHS images are semi-automatically processed [2] to detect the contour of both the lumen wall and the external elastic membrane. Only images containing the R-peak marker are selected, thus restricting analysis to the end-diastolic shape of the 
artery. The $X, Y$ positions of the detected points are transferred to a file, together with the $R-R$ interval. The time at which each image was recorded is interpreted as $\mathrm{Z}$-axis value, making use of the fact that the intravascular ultrasound catheter moved at constant speed through the artery.

Using MATLAB, a numeric computation and visualization program, the collected points are resampled at a constant $Z$-axis interval of 1 second, which translates into $1 \mathrm{~mm}$ distances between successive cross-sectional images. The contour data are subsequently converted to cylindrical coordinates, thus expressing the vessel wall, as well as the external elastic membrane by $r=f(\varphi, z)$, where $\mathrm{r}=$ radius $; \varphi=$ angle and $\mathrm{z}=$ position along $\mathrm{z}$-axis. Next a smoothing algorithm is applied to the surface $f(\varphi, z)$ by removing the higher frequency components of its 2D Fourier transform. The final result is a stack of IVUS cross-sectional images (Figure 1), all aligned perpendicular to and centered around the $Z$-axis.

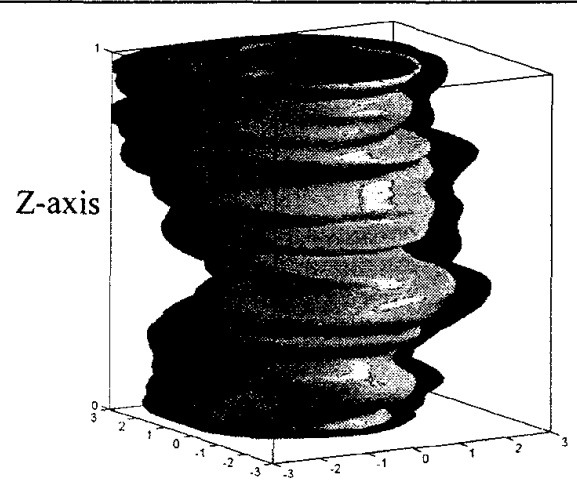

Figure 1: 3D View of stack of smoothed IVUS crosssections (Z-axis not to scale). Dark surface: external elastic membrane, light surface: vessel lumen.

\subsection{Reconstruction of the centerline}

In order to achieve a true $3 \mathrm{D}$ reconstruction of the shape of the vessel, we need to assess the real 3D path of the IVUS catheter tip. In order to do so, we produce two pictures, showing both of the biplane X-ray images with the position of the IVUS catheter at the start of the pull back procedure. As stated before, we may assume that the trajectory projections followed by the catheter tip closely coincide with the centerline of the catheter image in its biplane projections.

An alternative to this method would be to record the trajectory of the tip by filming its position many times during pull back. An investigation of this method showed that a considerable amount of additional movement is superimposed on the catheter path, even when gating for respiration and heart contraction. Therefor, we rejected this method because it lacks the required accuracy.

After digitization of the biplane projections using a custom build program, MATLAB routines are used to perform the necessary calculations to reconstruct the true 3D path of the catheter from its biplane shadow images. A first $3 \mathrm{D}$ guess is described in Fourier parameter form. Using an independent variable $(0 \geq \mathbf{s} \geq 1)$ the catheter centerline $\mathbf{R}(\mathbf{s})$ can be described by:

$$
\begin{aligned}
\bar{R}(s)=\bar{a}+ & \bar{b} \cdot s+\bar{c} \cdot \sin (2 \pi \cdot s)+\bar{d} \cdot \cos (2 \pi \cdot s)+ \\
& \bar{e} \cdot \sin (4 \pi . s)+\bar{f} \cdot \cos (4 \pi . s)+\ldots \ldots \ldots
\end{aligned}
$$

Using a Gauss-Newton optimization method the parameter vectors $\overline{\boldsymbol{a}}, \overline{\boldsymbol{b}}, \overline{\boldsymbol{c}}, \overline{\boldsymbol{d}}, \overline{\boldsymbol{e}}, \overline{\boldsymbol{f}} \ldots$ are adjusted, such that the projection of the 'guessed' 3D curve onto each of the image intensifier planes coincides as closely as possible, according to a minimum cost algorithm, with the filmed images of the IVUS catheter centerline.

Although it can be shown that not all possible curves can be reconstructed in this way, due to the existence of 'blind planes': planes that are perpendicular to both of the image intensifier entrance screens, with some caution and experience it is possible to select a constellation of both image intensifier planes, such that sections of the curve in the 'blind' position are avoided.

\subsection{Combination of angio and IVUS data}

After having acquired a stack of cross-sections as described in section 2.1 , and a 3D centerline as described in section 2.2, the stack has to be 'fitted' around the centerline. Because the start signal from the fluoroscopy film camera is recorded on the audio track of the IVUS VHS video, the position of the stack along the centerline is known. It is also known that the stack of crosssectional images contains pictures at $1 \mathrm{~mm}$ intervals, measured along the centerline. Two different unknowns however have still to be taken into account:

a) Under certain conditions, the catheter tip will exhibit a twist with respect to an external, fixed system of axes (Figure 2.).

b) The rotational position of the stack of images is unknown. 


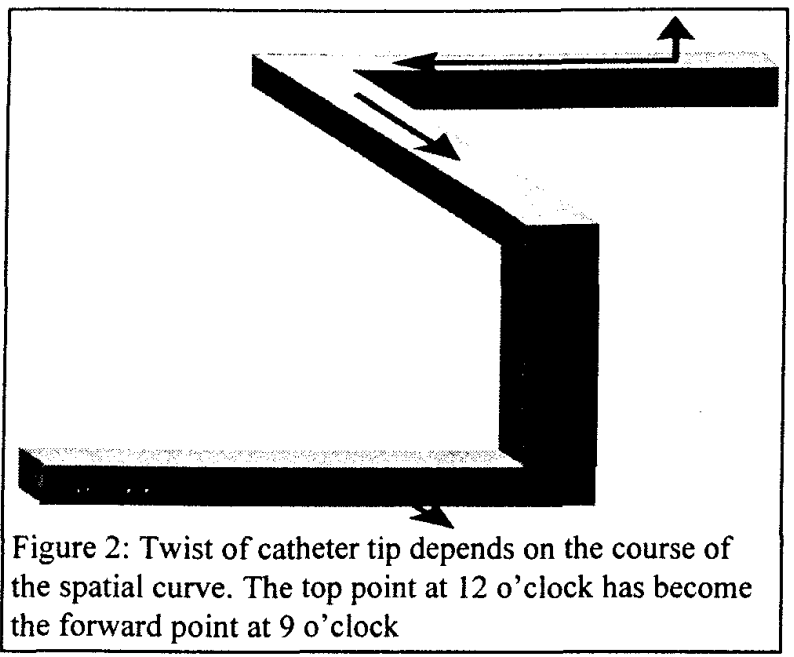

The effect under (a) can be explained as follows. If the catheter itself is assumed to be torsion free, the catheter tip will twist if the curve enters another plane. The amount of twist can be found from the Frenet formulas [4], from which it can be derived that the tip will rotate if the third derivative $\left(\mathrm{d}^{3} R / \mathrm{ds}^{3}\right)$ of the parametric curve $R(\mathrm{~s})$ does have a non-zero component in a direction perpendicular to both $T$ and $N$, where $T$ denotes the tangent $(T=\mathrm{d} R / \mathrm{ds}$ ) to the curve $R(\mathrm{~s})$ and $N$ denotes the normal $(N=\mathrm{d} T / \mathrm{ds})$ to the curve $\boldsymbol{R}$. Knowing this, one can devise an algorithm that takes this relative rotation i.e. the rotation from one cross-sectional image to the next, into account. The algorithm we use to reconstruct the 3D vessel by wrapping successive cross-sectional IVUS images around the reconstructed $3 \mathrm{D}$ catheter centerline, takes the relative rotational differences between successive cross-sections into account by considering the change in local radius of the computed catheter centerline.

This finally leaves us with the problem mentioned under (b). Although the rotation of each vessel crosssection is known with respect to the former one, the rotational position of the first cross-section remains unknown. If we change the rotational position of the first cross-sectional image around the catheter centerline, the whole of the vessel wall will rotate around the fixed and curved centerline. We refer to this phenomenon as 'sock rotation', because of the similarity of the situation with a sock on a leg.

In order to assess the correct sock rotation, the reconstruction of the $3 \mathrm{D}$ vessel wall is computed for all sock rotations between $0^{\circ}$ and $360^{\circ}$ in $10^{\circ}$ steps. The two biplane projections of these $3 \mathrm{D}$ reconstructions are then computed resulting in 36 reconstructed biplane frames. While single stepping forward and backward through this sequence of pictures a visual comparison is made between each of the computed pictures and the biplane angio as recorded at the end of the pull back procedure. Because real vessels always exhibit landmarks in the form of irregularities, side branches and bends, the most suitable

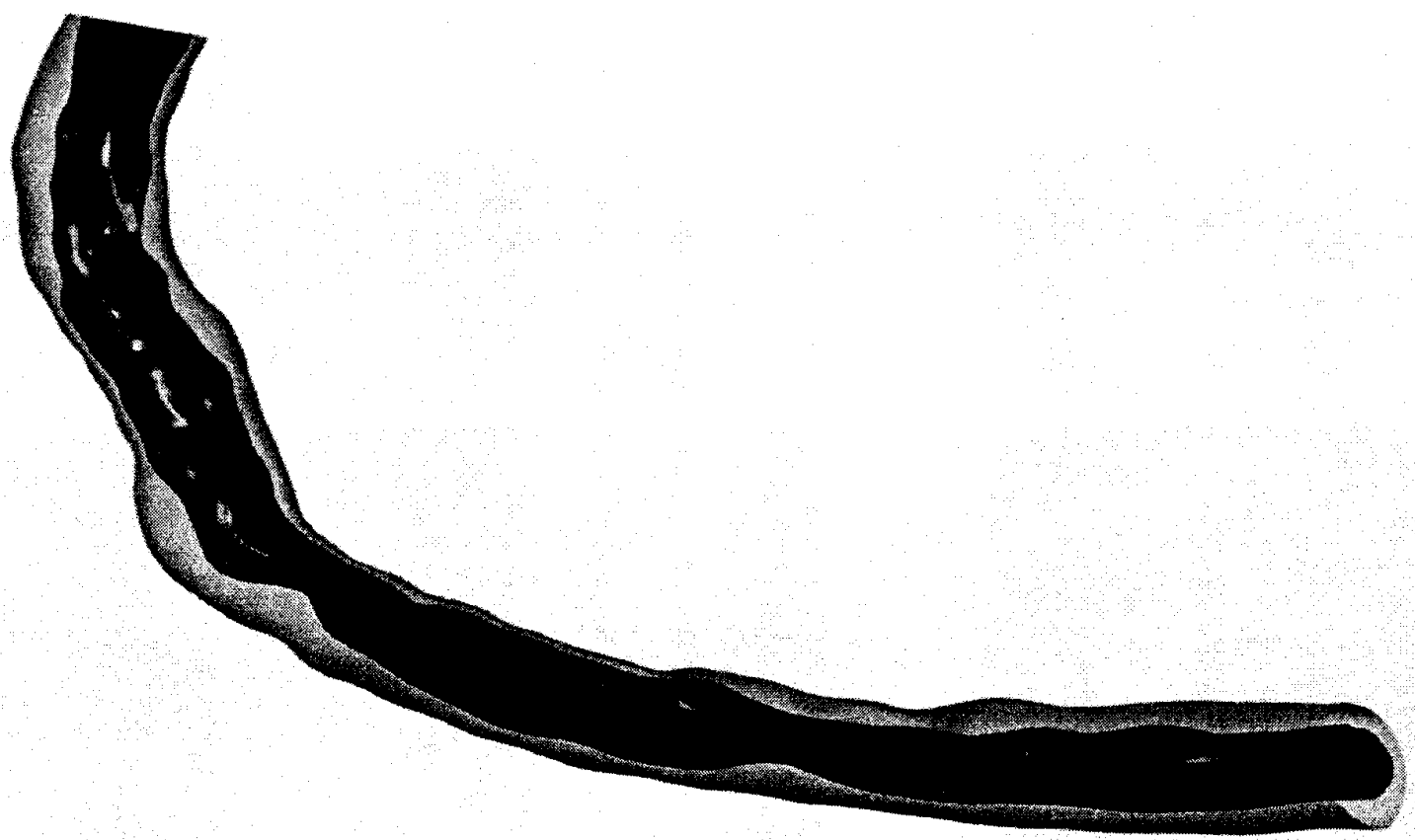

Figure 3: Rendering of a reconstructed in-vivo vessel. 
sock rotation can be estimated from this procedure.

A final computation is then made to arrive at a full $3 \mathrm{D}$ reconstruction of the vessel.

\section{Results}

In order to assess the accuracy of the method described here, a phantom has been constructed, consisting of a plastic cylinder with a diameter of 120 $\mathrm{mm}$, into which we cut a helical groove, with a width of 3 $\mathrm{mm}$ and a depth of $3 \mathrm{~mm}$. The bottom of the groove is semicircular. The pitch of the groove is $100 \mathrm{~mm}$ for one complete 360 degree turn. The groove was covered with a watertight flat plastic ribbon acting as a lid. Because of the rounded bottom and flat lid, the groove exhibits a Dshaped cross-section. The groove was filled with X-ray contrast medium and the afore mentioned protocol was followed to obtain the IVUS images and the biplane X-ray pictures from this phantom.

Studying the trajectory within this phantom, a high accuracy was found, with a distance between real and reconstructed trajectory $<1 \mathrm{~mm}$. First application in a human right coronary artery in-vivo (length: $75 \mathrm{~mm}$ ) showed a difference of $2 \%$ between the length of the reconstructed trajectory and the pull-back distance. Location and shape of stenoses in simulated projections of the $3 \mathrm{D}$ reconstructed lumen matched well with biplane angiography (Figure 3).

\section{Discussion}

The method as described here requires a high degree of accuracy from the biplane X-ray installation. We found that errors like non-coinciding isocenters (distance $\approx 2$ $\mathrm{mm})$ and a rotation of the image by the intensifier $\left(\approx 3^{\circ}\right)$ influenced the results noticeably. Nevertheless, due to the best fit nature of the minimum cost algorithm that is used, we achieved satisfying results with our first clinical trials.

The advantage of the described method above the common cylindrical straight reconstruction, can be appreciated if one considers for instance the problem of estimating the extent of plaque material. Although IVUS will allow an estimation of the thickness of the plaque material, when calculating a volumetric estimate of sclerotic material, one should take the position of the plaque relative to the bend of the vessel into account. (Figure 4).

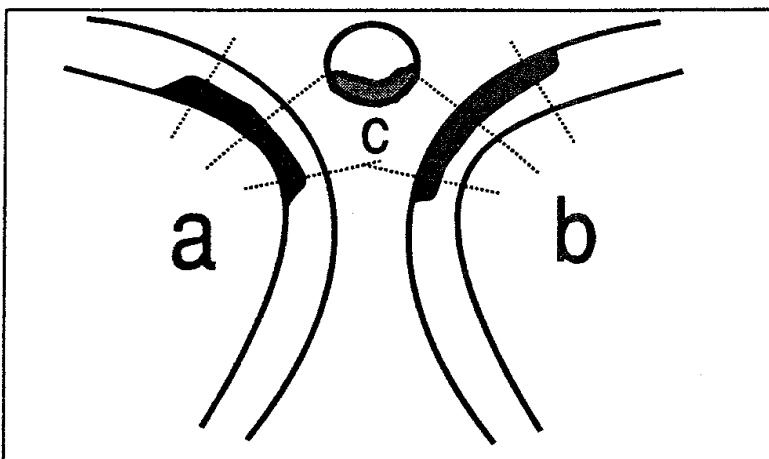

Figure 4: The position of a sclerotic plaque in a inside bend (a) or outside bend (b) influences its extent, without a marked change in IVUS image (c).

This reconstruction method might also find use in studies where one attempts to reconstruct the flow pattern in diseased arteries using finite element analysis.

\section{References}

[1] Slager CJ, Laban M, von Birgelen C, Krams R, Oomen JA, den Boer A, Li W, de Feyter PJ, Serruys PW, Roelandt JRTC. "ANGUS: A new approach to three-dimensional reconstruction of geometry and orientation of coronary lumen and plaque by combined use of coronary angiography and IVUS". J Am Coll Cardiol 1995;25:144A.

[2] Roelandt JRTC, Dimario C, Pandian NG, Li W, Keane D, Slager CJ, de Feyter PJ, Serruys PW. "Three dimensional reconstruction of intracoronary ultrasound images - Rationale, approaches, problems and directions" Circ 90:2, 1994, 10441055.

[3] Li W, Bosch JG, Zongh Y, van Urk H, Gussenhoven EJ, Mastik F, van Egmond F, Rijsterborgh H, Reiber JCH, Bom N. "Image segmentation and 3D reconstruction of intravascular ultrasound images". In: "Acoustical Imaging" ed. Y.Wei and B.Gu. Plenum Press, New York 1993.

[4] Davis HF, Snider AD. Introduction to Vector Analysis. Wm.C.Brown Publishers, 1988, 86:88.

Address for correspondence:

J.A. Oomen M.Sc.

Room EE 2322

Erasmus Univerity

PB 1738

3000 DR Rotterdam - The Netherlands

oomen@tch.fgg.eur.nl 